\title{
Validation of the Measurement of the Angle of Trunk Rotation in Photogrammetry
}

\author{
Isis J.R.L. Navarro, MSc, Cláudia T. Candotti, PhD, Maiane A. do Amaral, PT, Vinícius H. Dutra, BS,
} Grazielle M. Gelain, BS, and Jefferson F. Loss, PhD

ABSTRACT

\begin{abstract}
Objective: The purpose of this study was to validate the photogrammetric measurement of the angle of trunk rotation in relation to the scoliometer instrument.

Methods: Fifty-eight prominences from individuals with ages between 7 and 18 and with suspicion of spinal asymmetry (SA) were evaluated through the scoliometer and photogrammetry. The photographs were analyzed in the Digital Image-based Postural Assessment software. For statistical purposes, we used Pearson's correlation test (r), root mean square error, Bland-Altman graphical analysis, and receiver operating characteristic curve. The level of significance was $P \leq .05$.

Results: Excellent correlation for the angle of trunk rotation was obtained between the scoliometer and photogrammetry, with a root mean square error of $3^{\circ}$. The Bland-Altman graphical analysis showed equally dispersed data with no participants outside the limits of agreement. The receiver operating characteristic curve evidenced that (1) the cutoff point for the identification of the presence of spinal asymmetry is $4^{\circ} ;(2)$ mild to moderate SA is between $4^{\circ}$ and $7^{\circ}$; (3) moderate to severe SA is above $8^{\circ}$; and (4) sensitivity and specificity were above $83 \%$ and $78 \%$, respectively, with an area under the curve $\geq 90 \%$.

Conclusion: Photogrammetry is validated for measuring the angle of trunk rotation, being an accurate and accessible tool for the evaluation of patients with spinal asymmetries. (J Manipulative Physiol Ther 2020;43:50-56)

Key Indexing Terms: Scoliosis; Validation Studies; Photogrammetry; Posture; Evaluation Studies
\end{abstract}

\section{INTRODUCTION}

Idiopathic scoliosis (IS) is a 3-dimensional deformity of the spine and trunk, characterized by the distortion of the spine in 1 or more curves in the frontal plane; alteration of the alignment in the sagittal plane; and axial rotation of the vertebrae, and consequently of the trunk, in the transverse plane. ${ }^{1}$ The rotation of the trunk is evidenced in the thoracic spine by the posterior protuberance of the ribs in the region of the convexity of the curve. In clinical practice, spinal asymmetry (SA) is often linked with scoliosis. The Adams test, in which the participant is required to bend the trunk forward, is traditionally used to reveal the prominence. ${ }^{2-4}$

Among the instruments to evaluate the rotational component of SA, we have computerized tomography and

Biomechanics Department, Universidade Federal do Rio Grande do Sul, Porto Alegre, Rio Grande do Sul, Brazil.

Corresponding author: Isis J. R. L. Navarro, BS, ESEFID/ LAPEX/BIOMEC, Rua Felizardo, 750 Porto Alegre/RS Brazil CEP 90690-200.

(e-mail: isisjlnavarro@gmail.com).

Paper submitted November 19, 2018; in revised form April

30, 2019; accepted May 1, 2019.

0161-4754

(C) 2020 by National University of Health Sciences.

https://doi.org/10.1016/j.jmpt.2019.05.005 radiography, although these options are considered highcost and invasive exams. ${ }^{5-9}$ An alternative to these exams is the scoliometer, a valid and reproducible device, with well-defined cutoff points, designed to measure the asymmetry in the transverse plane of individuals with scoliosis. ${ }^{10}$ Also, the use of smartphones for this purpose has been suggested, but the verification of their sensitivity and specificity demands more studies. ${ }^{11-14}$

Another option to measure the angle of trunk rotation (ATR) is the photogrammetric analysis, which has been extensively used by researchers and health professionals. ${ }^{15,16}$ The photogrammetric analysis requires dedicated software and more complex procedures to provide the measurements, whereas the scoliometer has a direct and practical measure. However, considering the 3-dimensional characteristics of the spinal asymmetries linked with scoliosis, the use of photogrammetry could offer information about all planes in a single instrument. Nevertheless, as far as we know, there is still scarce information regarding the validity and reliability of photogrammetry in the transverse plane, and no cutoff points have been defined for SA.

Thus, the objective of the present study was to validate the photogrammetric measurement of the ATR in relation to the scoliometer instrument. We hypothesized that 

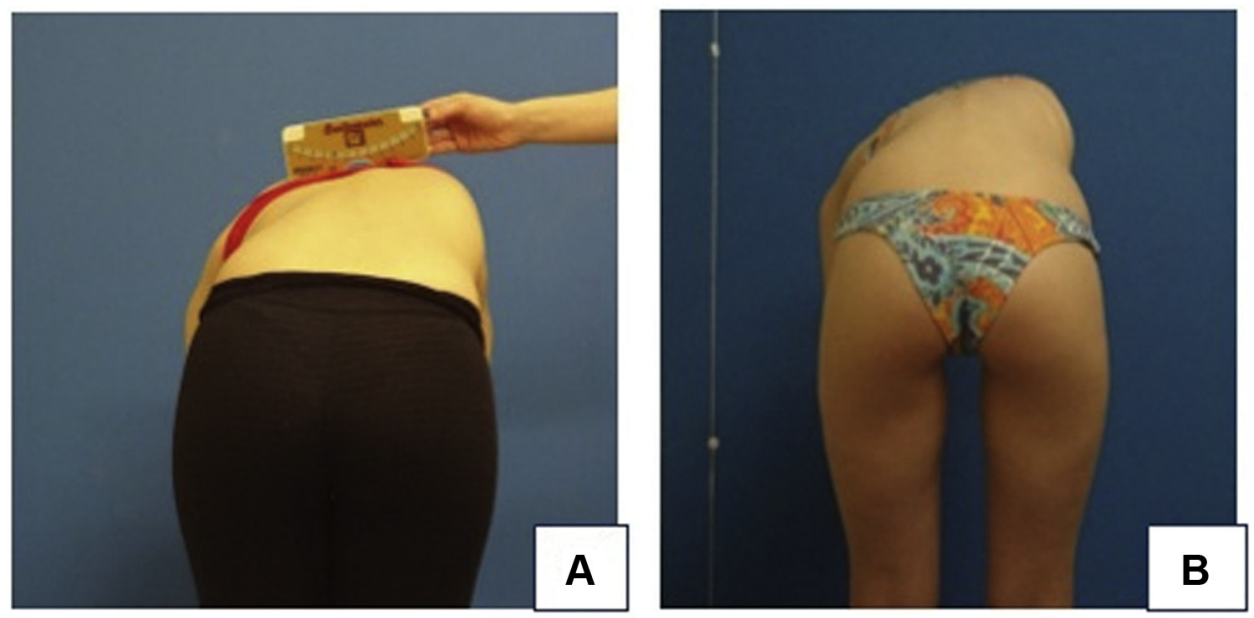

Fig I. Procedures for obtaining the angle of trunk rotation using (A) the scoliometer and (B) photogrammetry.

photogrammetry is capable of measuring the angle of trunk rotation analogously to the scoliometer.

\section{MeTHODS}

\section{Study Design}

This was a cross-sectional study for diagnostic test assessment that followed the Standards for Reporting Diagnostic Accuracy. ${ }^{17}$

\section{Participants}

We included consecutive participants of both biological sexes. Eligibility criteria included age between 7 and 18 years old, ability to maintain standing position without help, and leg length discrepancy less than $1.5 \mathrm{~cm}$. Participants were excluded if they had undergone surgical intervention of the spine. Sample size was calculated on G*Power 3.1.9.2, using a bivariate normal model correlation test (exact family test), power of $95 \%$, and probabilistic error of $5 \%$ considering $\mathrm{H}_{0} \mathrm{r}=0.4$ and $\mathrm{H}_{1} \mathrm{r}=0.7$, resulting in a sample of 58 prominences. An extra prominence was added in case of sample loss.

Ethical approval was granted by the Comitê de Ética em Pesquisa da UFRGS (UFRGS Research Ethics Committee) (CAAE 66785817.4.0000.5347). Informed consent was obtained from all individual participants included in the study. The individuals participated if they had agreed to be evaluated and only once their parents had signed the informed consent form before the assessments. The participants were identified through social network communication (Facebook, blog, WhatsApp) between June 2017 and April 2018.

\section{Test Methods}

The participants were submitted to 2 evaluation procedures. The order of the evaluation was random, but they were performed consecutively by the same evaluator.
For examination using the scoliometer, the participant was instructed to forward bend the trunk (Adams test) ${ }^{3,4}$ while the examiner glided the scoliometer on the back surface, keeping the spinous processes touching the cavity indicated in the instrument. From this procedure, it is possible to observe the clinical sign of prominence. The ATR was obtained from the regions of evidence (Fig 1A). Furthermore, the examiner identified the vertebral level where the clinical sign was found. The procedure was performed 3 times, and the larger ATR was considered. If a participant presented 2 prominences, both were evaluated.

Scoliometer measuring of the ATR has been described in the literature as a useful parameter for screening and early detection of spinal deformities. ${ }^{2,10,18}$ This very common tool is used among researchers and clinicians. Its cutoff point is usually determined between $5^{\circ}$ and $7^{\circ} \cdot{ }^{19,20}$ Thus, we considered the spinal asymmetry when the ATR was greater than $5^{\circ}$.

For examination using photogrammetry, the photograph was taken in the Adams test position, when the apex of the prominence was in evidence for the camera lens. Similar to the scoliometer examination, if a participant presented 2 prominences, both were evaluated. A plumb line with 2 markers was parallel to the external malleolus. The digital camera (Sony Cyber-Shot DSC-F717, 5.0 megapixels, 512 MB of memory, $5 \times$ optical zoom and $10 \times$ digital zoom) was attached to a tripod at a .95-cm height and $3.0 \mathrm{~m}$ horizontally distant from the individual. The distances were previously checked considering zoom options and proportionality of the original image ${ }^{16}$ (Fig 1B). The photographs were digitalized and analyzed on the Digital Image-based Postural Assessment (DIPA) software.

\section{Procedure for Image Analysis}

The images were digitalized on the DIPA software by the same evaluator, who was not the same person who was 


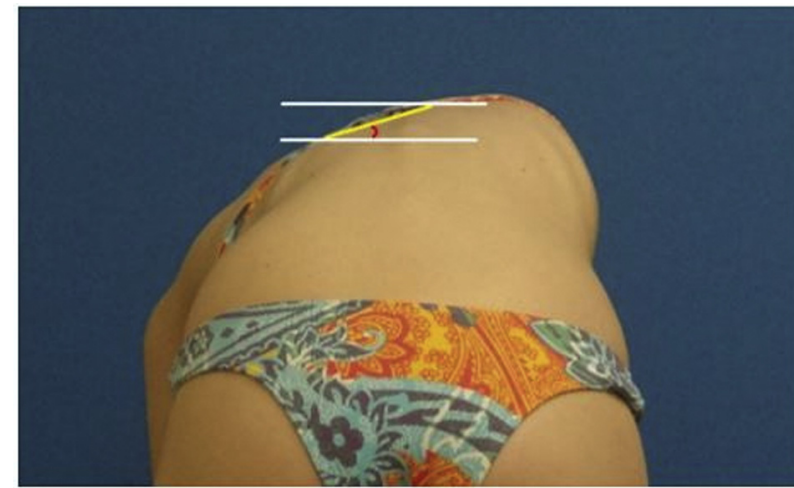

Fig 2. The photogrammetric image shows the straight line connecting the selected points (yellow line) and the parallel lines (white lines).

responsible for and performed the data collection. Two markers on the plumb line represented the spatial reference for the software. Two points representing the superior apexes of the prominence nearer the vertebrae in each half-body, left and right, respectively, were digitalized in the photography. The location of these 2 points makes the measurement of the ATR the most similar one to the scoliometer. The software draws a straight line between the digitized points on the half-body and another 2 horizontal lines, which were parallel to the ground. Each parallel line passed on one of the selected points (Fig 2). The angle formed by the line between the digitized points and the respective parallel lines indicates the magnitude of the ATR on the body surface. There are no established cutoff point values for the ATR on photogrammetry. Based on the findings of this research, we might suggest a new way to perform this measure and some cutoff points for the angle of trunk rotation measured by means of photogrammetry.

For further statistical analysis, the angles provided by photogrammetry were transformed using a simple linear regression analysis. The data were plotted in a scatter diagram (scoliometer vs photogrammetry) and the regression line (minimum square method) was used $\left(\mathrm{R}^{2}=0.92\right)$ to transform the angles obtained on photogrammetry: $\mathrm{y}=$ $0.94 \mathrm{x}+0.70$, where $\mathrm{x}$ represents the originally measured angles on photogrammetry and y represents the transformed angles, which were later used in the statistical analysis. The normality was verified through the analysis of the residue histogram, the linearity through the P-P plot of regression standardized residual, the homoscedasticity through the visual analysis of the scatterplot (residual vs predicted), and the independency of residuals through the analysis of the Durbin-Watson value (1.739).

\section{Statistical Analysis}

There were no indeterminate results or missing data for both scoliometer and photogrammetry evaluations. The data were analyzed using the Statistical Package for the Social Sciences version 21.0. The outliers were identified based on the analysis of residual statistics (Statistical Package for the Social Sciences) and they were excluded. The validity was appraised using (1) Pearson's correlation coefficient, (2) root mean square (RMS) error, and (3) Bland-Altman (B\&A) plot analysis. The correlation was classified according to $\mathrm{Cohen}^{21}$ as weak (0.10-0.29), moderate $(0.30-0.49)$, and excellent $(0.50-1)$. The receiver operating characteristic (ROC) curve was used to determine the diagnostic accuracy and to establish the cutoff point for classifying spinal asymmetries using photogrammetry. The area under the curve (AUC) was classified according to Hanley and $\mathrm{McNeil}^{22}$ as poor (0.60-0.69), regular (0.70-0.79), and excellent $(\geq 0.90)$. The significance level adopted was 0.05 .

\section{RESULTS}

\section{Participants}

The sample is described in the flow diagram (Fig 3). It was composed of 58 prominences from 53 participants. Two outlier participants were excluded, resulting in 51 participants. Five participants had 2 prominences, totaling 56 prominences analyzed. The participants were $64 \%$ female $(\mathrm{n}=34)$ and presented a mean age of $14 \pm 2.9$ years old, a mean body mass of $52 \pm 12.9 \mathrm{~kg}$, and a mean height of 1.60 $\pm 0.1 \mathrm{~m}$. The mean ATR obtained through the scoliometer and photogrammetry was $8^{\circ}\left(\operatorname{minimum} 0^{\circ}, \max 30^{\circ}\right)$ and $7^{\circ}$ (minimum $0^{\circ}$, maximum $27^{\circ}$ ), respectively.

The correlation between the measurements of both instruments was excellent, with an RMS error of $3^{\circ}$, and it was considered satisfactory (Table 1). In the B\&A plot analysis, the mean difference between the 2 instruments was $0^{\circ}$, with upper and lower limits between $5.6^{\circ}$ and $-5.6^{\circ}$, respectively. The data were scattered, with no individuals outside the upper or the lower limits (Fig 4).

The ROC curve showed that, in photogrammetry, the cutoff point of the ATR to identify the presence of spinal asymmetry is $4^{\circ}$ and that it is possible to classify the severity of spinal asymmetry. The ATR between $4^{\circ}$ and $7^{\circ}$ indicates mild to moderate spinal asymmetry, whereas the ATR above $8^{\circ}$ indicates moderate to severe spinal asymmetry. All cutoff points presented an excellent AUC $(\geq 90 \%)$ with sensitivity and specificity measures greater than $83 \%$ and $78 \%$, respectively (Table 2 ).

\section{DISCUSSION}

Our results showed high and significant correlation of the ATR and a small RMS error, demonstrating the reliability and accuracy of photogrammetry in the evaluation of the ATR. Considering that the correlation coefficient is a measure of association, which evaluates the relationship 


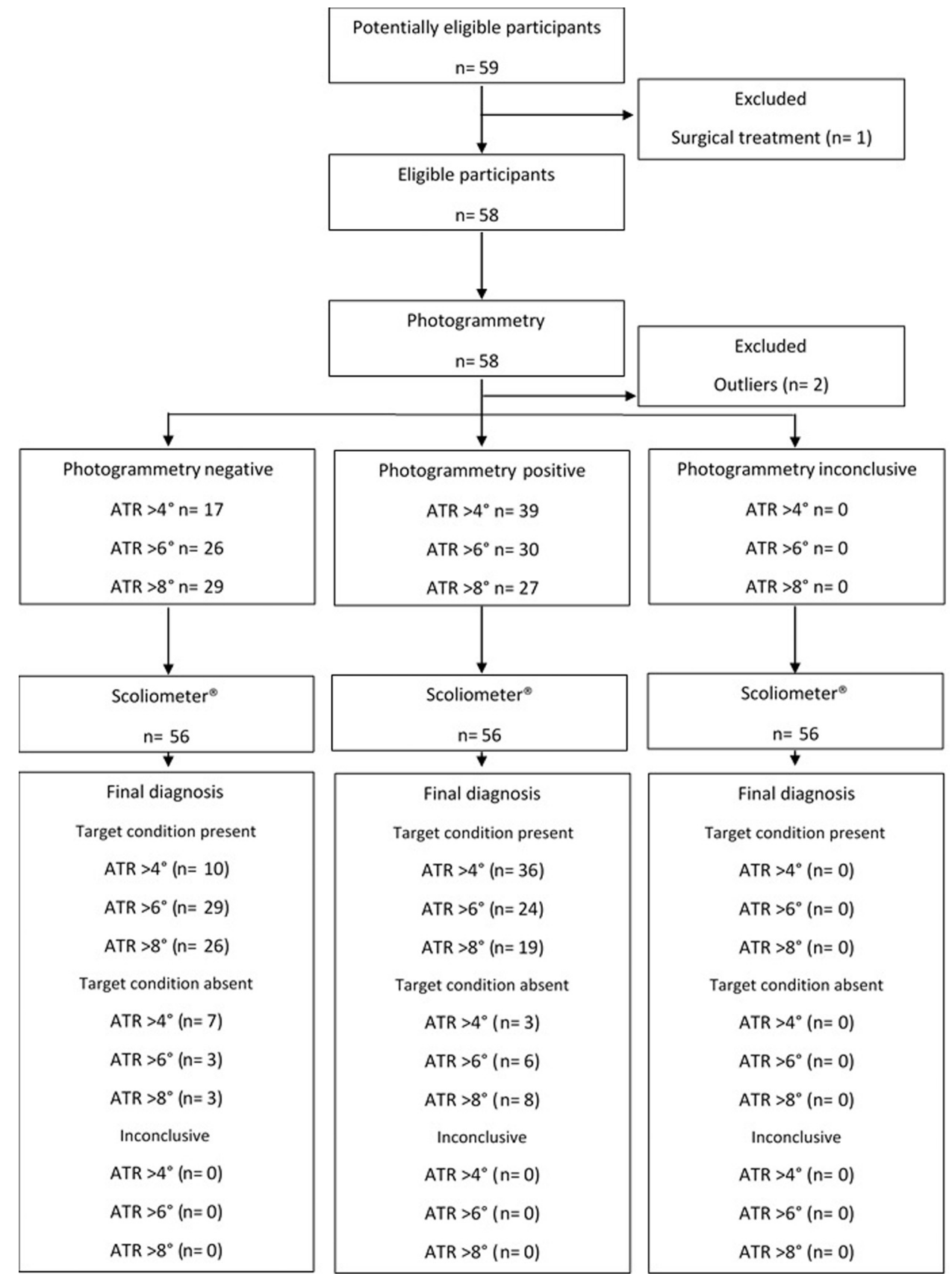

Fig 3. Flow diagram of the participants. ATR, angle of trunk rotation.

between 2 variables, but cannot show the differences between them, ${ }^{23}$ we have performed the B\&A plot analysis. This analysis describes and quantifies the agreement between 2 measures from a scatter plot in which the $y$ axis shows the difference between these measures and the $\mathrm{x}$ axis shows the mean of these measures. Moreover, the plot demonstrates the presence of outliers or trends between the measures. ${ }^{23-25}$

In this analysis, the ATR of photogrammetry and the scoliometer were dispersed and no individuals were outside the limits of agreement. This result, associated with the small RMS error, demonstrates the agreement between the 2 instruments and reinforces the confidence in the use of photogrammetry (DIPA software) to measure the ATR. The DIPA software was used in our study and has already been validated, besides being an accurate, accessible, and easy-to-handle tool for assessing the spine in sagittal and frontal planes. ${ }^{15,16}$ This software provides the angles of dorsal kyphosis and lumbar lordosis, as well as the spinal inclination angle in the frontal plane. 
Table I. Mean ATR Obtained From the Scoliometer and Photogrammetry, Correlation Coefficient, and RMS Error

\begin{tabular}{lllll}
\hline & Scoliometer Mean (min-max) & Photogrammetry Mean (min-max) & r & RMS Error \\
\hline ATR & $8^{\circ}(0-30)$ & $7^{\circ}(0-27)$ & .96 & $3^{\circ}$ \\
\hline$P<.05$
\end{tabular}

$P<.05$.

$A T R$, angle of trunk rotation; $\max$, maximal; $\min$, minimal; $r$, Pearson correlation coefficient; $R M S$, root mean square.

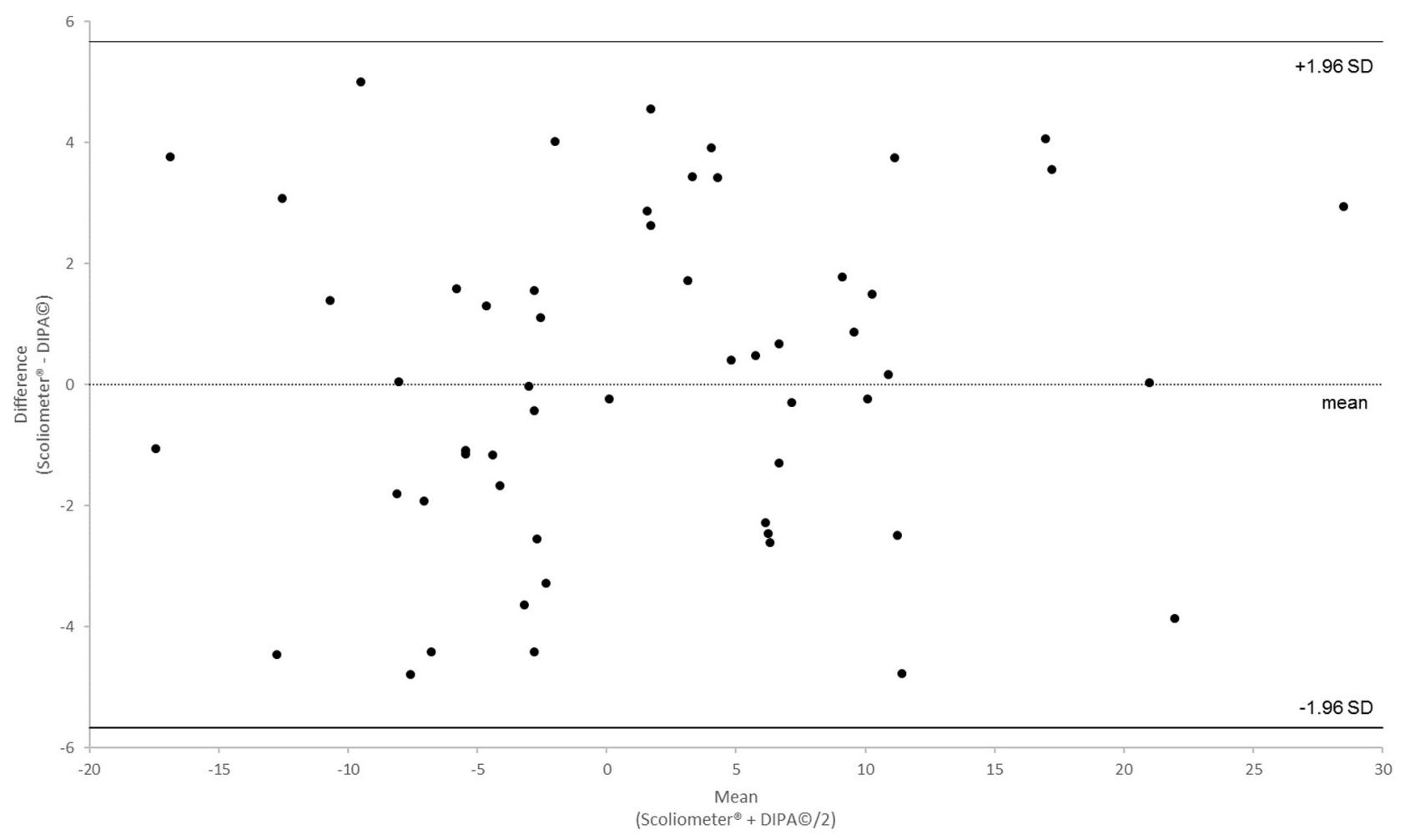

Fig 4. Bland-Altman plot analysis comparing scoliometer vs ATR in photogrammetry $\left({ }^{\circ}\right)$. DIPA, Digital Image-based Postural Assessment; SD, standard deviation.

With the increasing popularity of smartphones, some applications are being developed as an alternative to the scoliometer to reproduce the function of the instrument, with the same accuracy, to measure the ATR. ${ }^{11-14}$ However, the applications that presented validation or reproducibility are only available for the iOS operating system, restricting its usage. In addition, the limitation of those applications is the measure magnitude restricted to a maximum of $30^{\circ}$, as in the scoliometer, whereas the DIPA software does not have a limit of measure. Also, the sensitivity and specificity of those applications still demand more investigation.

The torsion bottle is a very simple tool to verify the presence of prominence associated with scoliosis and is an alternative to the scoliometer. Romano and Mastrantonio ${ }^{26}$ showed the torsion bottle is a reliable tool when compared with the scoliometer, even in the interrater analysis. Although a very simple and useful tool, it does not provide the magnitude of the ATR. Thus, the use of the torsion bottle is limited to basic screening, domestic use, or preinvestigation patients. Despite requiring a dedicated software and more complex procedures to provide the measurements, the DIPA software provides benefits like allowing the quantification of the ATR, getting an objective registry of patient's prominence, allowing professional use, and offering an unlimited range of the measures. Also, with a single instrument, it is possible to assess the 3 planes of scoliosis.

In the validation process of an instrument, it is important to know the probability of accurate results being provided. Sensitivity is the probability of a positive test in the presence of a disease (true positive), whereas specificity is the probability of a negative test in the absence of a disease (true negative). ${ }^{27}$ The ROC curve provides these measures through graphical representation. ${ }^{9}$ Our results showed that photogrammetry (DIPA software) can identify the presence of spinal asymmetry from the ATR measurement with a cutoff point of $4^{\circ}$ and with an excellent AUC (91\%). 
Table 2. Cutoff Point for Classifying the Scoliotic Curve Using Photogrammetry and Measures of Sensitivity and Specificity (ROC Curve)

\begin{tabular}{|c|c|c|c|c|c|}
\hline $\begin{array}{l}\text { Cutoff Point Using the } \\
\text { Scoliometer }\end{array}$ & $\begin{array}{l}\text { Cutoff Point Using } \\
\text { Photogrammetry }\end{array}$ & Sensitivity & Specificity & $\begin{array}{l}\text { Area Under } \\
\text { the Curve }\end{array}$ & CI $(95 \%)$ \\
\hline$>4^{\circ}$ & $\geq 3.7^{\circ}$ & $84 \%$ & $85 \%$ & $.91^{\mathrm{a}}$ & $83-98$ \\
\hline$>5^{\circ}$ & $\geq 4.7^{\circ}$ & $88 \%$ & $78 \%$ & $.90^{\mathrm{a}}$ & $82-98$ \\
\hline$>6^{\circ}$ & $\geq 5.8^{\circ}$ & $89 \%$ & $83 \%$ & $.94^{\mathrm{a}}$ & $88-100$ \\
\hline$>7^{\circ}$ & $\geq 6.9^{\circ}$ & $83 \%$ & $81 \%$ & $.92^{\mathrm{a}}$ & $84-99$ \\
\hline$>8^{\circ}$ & $\geq 6.9^{\circ}$ & $86 \%$ & $79 \%$ & $.92^{\mathrm{a}}$ & $84-100$ \\
\hline$>9^{\circ}$ & $\geq 8.1^{\circ}$ & $90 \%$ & $95 \%$ & $.92^{\mathrm{a}}$ & $84-100$ \\
\hline
\end{tabular}

$R O C$, receiver operating characteristic.

a $P \leq .001$.

It means that individuals presenting an ATR less than $4^{\circ}$ are classified as no spinal asymmetry. For this cutoff point, the sensitivity and specificity were $84 \%$ and $85 \%$, respectively, showing less than $15 \%$ probability of misdiagnosis. This result agrees with the cutoff point defined for diagnosis of spinal asymmetry through the scoliometer ${ }^{2,9,10}$ and demonstrates the clinical applicability of photogrammetry for the screening and early diagnosis in cases of spinal asymmetries, usually linked with scoliosis.

In addition, the severity of spinal asymmetry can also be identified. Individuals presenting an ATR wider than $8^{\circ}$ are classified as moderate/severe spinal asymmetry, with sensitivity and specificity of $90 \%$ and $95 \%$, respectively, and with an excellent AUC (92\%). This result, which corroborates Côte et al, ${ }^{9}$ has an important clinical relevance, since misdiagnosis in severe spinal asymmetry may be disadvantageous for the affected individual, considering the progression of the deformity. The specificity obtained indicates that photogrammetry is capable of identifying moderate or severe spinal asymmetry with a minimal proportion of possible false-positive results.

\section{Limitations}

The limitation of our study was the difficulty of evaluating the lumbar curves through photogrammetry, mainly owing to the lack of flexibility of the individuals. The stiffness of the hamstring muscles in many cases prevented the participant from reaching the flexion amplitude of the trunk required in the Adams test to assess the lumbar region. ${ }^{28}$ In fact, Zampier Ulbrich ${ }^{29}$ explains that the outbreak of pubertal growth, due to hormonal changes, generates a more pronounced growth in the long bones than in muscles and tendons, causing a temporary decrease in the flexibility indexes for adolescents. A second limitation was the definition of the apexes of the curves on the photographs in each half-body, which probably induced the variability of these measures. Nevertheless, Côte et al ${ }^{9}$ had the same difficulty in determining the apex of the curves that influenced the positioning of the instrument and thus produced variability in the readings of the scoliometer.

\section{Conclusion}

The results demonstrated the accuracy of photogrammetry (DIPA software) in the measurement of the angle of trunk rotation, with strong correlation and agreement with the scoliometer. Also, evidenced in the analysis of the ROC curve was the diagnostic capacity of photogrammetry in the identification of spinal asymmetry through the measurement of the ATR, with consistent measures of sensitivity $(>83 \%)$ and specificity (>78\%) and an excellent AUC $(\geq 90 \%)$. Based on these outcomes, the proposed method can differentiate among the absence of spinal asymmetry, mild to moderate spinal asymmetry, and moderate to severe spinal asymmetry. These results have great relevance for clinical practice, considering the ease of handling, the low cost, and the possibility of keeping a photographic record with quantitative measures for future feedback and reevaluation.

\section{Funding SOURCES AND CONFLICTS OF INTEREST}

Isis Navarro received a master's degree scholarship from $\mathrm{CNPq}$ and Maiane Amaral received an undergraduate degree scholarship from CNPq. No conflicts of interest were reported for this study.

\section{CONTRIBUTORSHIP INFORMATION}

Concept development (provided idea for the research): I.J.R.L.N., C.T.C.

Design (planned the methods to generate the results): I.J.R.L.N., C.T.C.

Supervision (provided oversight, responsible for organization and implementation, writing of the manuscript): I.J.R.L.N., C.T.C. 
Data collection/processing (responsible for experiments, patient management, organization, or reporting data): I.J.R.L.N., M.A.A.

Analysis/interpretation (responsible for statistical analysis, evaluation, and presentation of the results): I.J.R.L.N., V.H.D., M.A.A., J.F.L.

Literature search (performed the literature search): I.J.R.L.N., M.A.A., G.M.G.

Writing (responsible for writing a substantive part of the manuscript): I.J.R.L.N., M.A.A., G.M.G.

Critical review (revised manuscript for intellectual content, this does not relate to spelling and grammar checking): I.J.R.L.N., C.T.C.

\section{Practical Applications}

- This study contributes to the development of new noninvasive tools for the evaluation of adolescent IS.

- Photogrammetry was shown to be accurate and validated for measuring the ATR.

- The results allowed us to establish cutoff points for the classification of the severity of adolescent IS.

\section{REFERENCES}

1. Negrini S, Donzelli S, Aulisa AG, et al. 2016 SOSORT guidelines: orthopaedic and rehabilitation treatment of idiopathic scoliosis during growth. Scoliosis Spinal Disord. 2018; 13(1):3.

2. Amendt LE, Ause-Ellias KL, Eybers JL, Wadsworth CT, Nielsen DH, Weinstein SL. Validity and reliability testing of the Scoliometer. Phys Ther. 1990;70(2):108-117.

3. Fairbank J. Historical perspective: William Adams, the forward bending test, and the spine of Gideon Algernon Mantell. Spine (Phila Pa 1976). 2004;29(17):1953-1955.

4. Altaf F, Gibson A, Dannawi Z, Noordeen H. Adolescent idiopathic scoliosis. BMJ. 2013;346:f2508.

5. Yazici M, Acaroglu ER, Alanay A, Deviren V, Cila A, Surat A. Measurement of vertebral rotation in standing versus supine position in adolescent idiopathic scoliosis. J Pediatr Orthop. 2001;21(2):252-256.

6. Aaro S, Dahlborn M. Estimation of vertebral rotation and the spinal and rib cage deformity in scoliosis by computer tomography. Spine (Phila Pa 1976). 1981;6(5):460-467.

7. Drerup B. Principles of measurement of vertebral rotation from frontal projections of the pedicles. J Biomech. 1984; 17(12):923-935.

8. Richards BS. Measurement error in assessment of vertebral rotation using the Perdriolle torsionmeter. Spine (Phila $\mathrm{Pa}$ 1976). 1992:17(5):513-517.

9. Côté P, Kreitz BG, Cassidy JD, Dzus AK, Martel J. A study of the diagnostic accuracy and reliability of the Scoliometer and
Adam's forward bend test. Spine (Phila Pa 1976). 1998;23(7): 796-802; discussion 803.

10. Bunnell WP. An objective criterion for scoliosis screening J Bone Joint Surg Am. 1984;66(9):1381-1387.

11. Franko OI, Bray C, Newton PO. Validation of a scoliometer smartphone app to assess scoliosis. J Pediatr Orthop. 2012; 32(8):e72-e75.

12. Balg F, Juteau M, Theoret C, Svotelis A, Grenier G. Validity and reliability of the iPhone to measure rib hump in scoliosis. J Pediatr Orthop. 2014;34(8):774-779.

13. Izatt MT, Bateman GR, Adam CJ. Evaluation of the iPhone with an acrylic sleeve versus the Scoliometer for rib hump measurement in scoliosis. Scoliosis. 2012;7(1):14.

14. Qiao J, Xu L, Zhu Z, et al. Inter- and intraobserver reliability assessment of the axial trunk rotation: manual versus smartphone-aided measurement tools. BMC Musculoskelet Disord. 2014;15:343.

15. Furlanetto TS, Sedrez JA, Candotti CT, Loss JF. Photogrammetry as a tool for the postural evaluation of the spine: a systematic review. World J Orthop. 2016; 7(2):136.

16. Furlanetto TS, Candotti CT, Comerlato T, Loss JF. Validating a postural evaluation method developed using a Digital Image-based Postural Assessment (DIPA) software. Comput Methods Programs Biomed. 2012;108(1):203-212.

17. Bossuyt PM, Reitsma JB, Bruns DE, et al. STARD 2015: an updated list of essential items for reporting diagnostic accuracy studies. Clin Chem. 2015;61(12):1446-1452.

18. Beauséjour M, Goulet L, Parent S, et al. The effectiveness of scoliosis screening programs: methods for systematic review and expert panel recommendations formulation. Scoliosis. 2013;8(1):12.

19. Grivas TB, Wade MH, Negrini S, et al. SOSORT consensus paper: school screening for scoliosis. Where are we today? Scoliosis. 2007;2:17.

20. Huang S-C. Cut-off point of the Scoliometer in school scoliosis screening. Spine (Phila Pa 1976). 1997;22(17): 1985-1989.

21. Cohen Jacob. Statistical Power Analysis for the Behavioral Sciences. 2nd ed. Hillsdale, NJ: Lawrence Erlbaum Associates; 1988.

22. Hanley JA, McNeil BJ. The meaning and use of the area under a receiver operating characteristic (ROC) curve. Radiology. 1982;143(1):29-36.

23. Giavarina D. Understanding Bland Altman analysis. Biochem Med (Zagreb). 2015;25(2):141-151.

24. Hirakata VN, Camey S. Análise de concordância entre métodos de Bland-Altman. Clin Biomed Res. 2009;29:261268.

25. Altman DG, Bland JM. Measurement in medicine: the analysis of method comparison studies. J R Stat Soc Ser D Stat. 1983;32(3):307-317.

26. Romano M, Mastrantonio M. Torsion bottle, a very simple, reliable, and cheap tool for a basic scoliosis screening. Scoliosis Spinal Disord. 2018;13:4.

27. Kawamura T. Interpretação de um teste sob a visão epidemiológica: eficiência de um teste. Arq Bras Cardiol. 2002;79(4).

28. de Araújo CGS. Avaliação da flexibilidade: valores normativos do flexiteste dos 5 aos 91 anos de idade. Arq Bras Cardiol. 2008;90(4).

29. Zampier Ulbrich A, Bozza R, Machado HS, et al. Aptidão física em crianças e adolescentes de diferentes estágios maturacionais. Fit Perform J. 2007;6(5):277-282. 